\title{
Numerical Study of the Effect of Dental Implant Inclination
}

\author{
D. Šarac ${ }^{1}$, I. Atanasovska ${ }^{2 *}$, S. Vulović ${ }^{3}$, N. Mitrović ${ }^{1}$, I. Tanasić ${ }^{4}$ \\ ${ }^{1}$ University of Belgrade, Faculty of Mechanical Engineering, 27 Kraljice Marije Street, 11000 \\ Belgrade, Serbia \\ e-mail: saracdusan1986@gmail.com \\ e-mail:nmitrovic@mas.bg.ac.rs \\ ${ }^{2}$ Mathematical Institute of the Serbian Academy of Sciences and Arts, 36 Kneza Mihaila Street, \\ 11000 Belgrade, Serbia \\ e-mail: iatanasovska@mi.sanu.ac.rs \\ ${ }^{3}$ Faculty of Engineering, University of Kragujevac,6 Sestre Janjic Street, 34000 Kragujevac, \\ Serbia \\ e-mail:vsneza@kg.ac.rs \\ ${ }^{4}$ University of Belgrade, School of Dentistry, Clinic of Prosthodontics, 4 Rankeova Street, \\ 11000 Belgrade, Serbia \\ e-mail: doktorivan@hotmail.com \\ *corresponding author
}

\begin{abstract}
This paper contains the results of a research focused on the determination of the influence of an implant inclination on the strain state throughout the acrylic block with implant. The aim of the presented research is to qualitatively determine the regions with the greatest strain fields on the models. The finite element models of implant and acrylic block are developed for predefined implant inclinations in order to analyze the influence of implant inclination on deformations on the outer surface of acrylic block. The comparative contour plots of stress and strain state of analyzed models, as well as the comparative diagrams with obtained results, are presented. The conclusions about the inclination angle which leads to the higher strains in the block-implant are explained. Obtained results could be applied for the planning of future experimental studies which could utilize this and similar models to determine their load transfer characteristics, and could be included in the planning of dental implant position, and prediction of successful dental therapy.
\end{abstract}

Keywords: Implant, inclination, FEA

\section{Introduction}

In order to minimize the value of bending moments and achieve optimal distribution, implants should be placed in vertical position, parallel with axial forces and occlusal plane (Mitrovic et al. 2016, Brown and Payne 2011, Behnaz et al. 2015, Gul and Suca 2014, Markarian and Ueda 2007). This is not always possible due to alveolar deficiency (Tian et al. 2012) or anatomical limitations (Markarian \& Ueda 2007, Watanabe et al. 2003). Also, in dental practice, it is 
known that implant inclination occurs, although vertical position was desired. According to Payer et al. (2008), this error most often occurs due to surgeons' inexperience.

Implant inclination has a significant role in biomechanics, and can influence the longevity of the treatment, following Gul and Suca (2014). The impact of implant inclination on the surrounding structure has been frequently researched in the overdenture settings (Behnaz et al. 2015, Gul and Suca 2014, Markarian and Ueda 2007, Cağlar et al. 2006, Almeida et al. 2013, Hong et al. 2012, Lan et al. 2010, Sljivic et al. 2016, Takahashi, Shimamura and Sakurai 2010, Ueda et al. 2004, Naini et al. 2011), rather than in an isolated environment, as explained in Watanabe et al. (2003). The intention in this study was to focus on the simpler loading conditions and to eliminate other systematical factors which arise due to the system complexity. Experimental stress and strain measurements of the human jaw and various models in the vicinity of the dental implant has been found in the literature (Markarian and Ueda 2007, Tanasic et al. 2012a, Tanasic et al. 2012b). 3D Finite element method (FEA) is an algebrabased computer simulation technique which has also been established as a reliable tool for analysis of different models.

Recently published studies used digital image correlation (Mitrovic N. 2011, McCormick, Lord 2010) to measure strain on the polymethil-metacrilate block, during axial loading of an embedded dental implant, as an attempt of the reference model standardization (Mitrovic et al. 2016, Tanasić et al. 2015). Our study was guided by this experimental research explain in Mitrovic et al. (2016) and Tanasic et al. (2015), and therefore utilized its model type and characteristics. Similarly, results are displayed in the form of strain. This analysis represents another step for the ongoing experimental study. Additionally, Tiossi et al. (2012) described how PMMA models could be used to determine the influence of prostheses design on the implants survival rate, due to their mechanical characteristics similar to bone presented by Tanasic et al. (2015). Therefore, it is considered that common models could provide better insight into biomechanics of the load transfer of the inclined implants.

The purpose of this paper is to determine and compare the impact of an implant inclination on the strain of the acrylic block and to qualitatively determine the regions of the greatest strain fields on the models.

\section{Numerical model developing and verification}

Three-dimensional Finite element (FE) model of implant and acrylic block is developed using commercial software ANSYS 13.0. Structural solid three-dimensional degenerate brick elements without midside nodes are used for implant and acrylic block material simulation. The contact surfaces between the implant and the acrylic block, in which the implant is poured, is defined as inherent and frictionless. All dimensions of the FE model are identical to the dimensions of the model used for experiment, as explained in Mitrovic et al. (2016). Figure 1a shows the three-dimensional FE model consisting of 259470 elements and 347255 nodes developed in accordance with the model used for experiment verification.

Two material models are used in this FE model - the first for the acrylic material and the second for the implant material. Boundary conditions and external load are defined thus to correspond to the supports and loads used during the experimental measurement. The external static load of $500 \mathrm{~N}$ is defined in the opposite $y$ direction and the appropriate displacement constraints are defined as shown in Figure $1 \mathrm{~b}$ on the acrylic block. The static Finite Element Analysis (FEA) is performed and the obtained strains interval is within the elasticity of the modeled materials. 
A two-dimensional FE model is then developed in order to make the FEA which needs less computer time. Structural solid two-dimensional quadrilateral plane finite elements with midside nodes are used for the implant and acrylic block plane model developing. The axial symmetrical option is used for 2D model generation.

The obtained Elastic Equivalent Von Mises strains are shown in Figure 2a as contour plots for the whole FE model (the implant and the acrylic block) and only for the acrylic block in Figure $2 \mathrm{~b}$. The elastic equivalent strains distribution has the expected layout. The cross section plot shown in Figure 3 is obtained by plane cutting that passes through the implant center and is parallel with $x y$ coordinate plane.
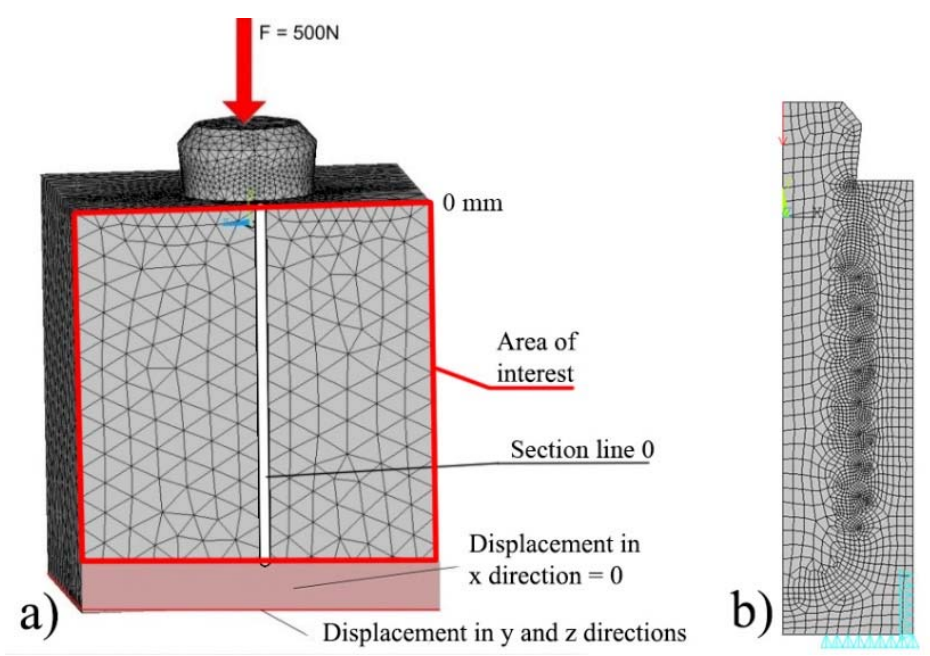

Fig. 1. a) FE model with constraints and external load definition - the case without implant inclination; b) Mesh density in the block-implant interface
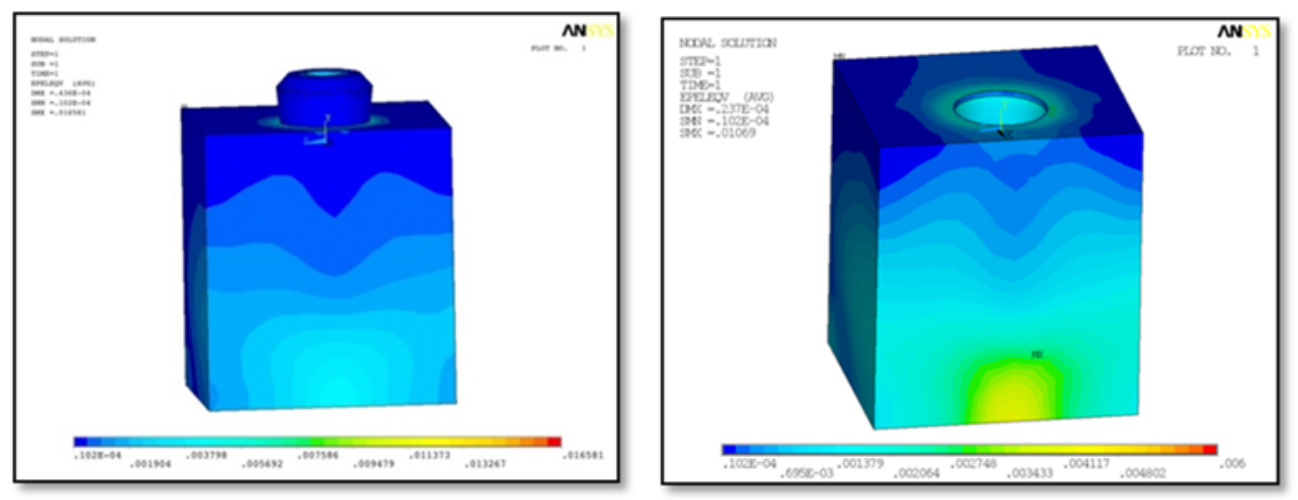

Fig. 2. Elastic Equivalent Von Mises strains. (a) Implant and acrylic block; (b) Acrylic block

The obtained Elastic Von Mises strains are shown in Fig. 4a as contour plots for the whole FE model (the implant and the acrylic block) and only for the acrylic block in Fig. 4b. High result matching obtained with the two-dimensional and three-dimensional simulation is more obvious when the results shown in Fig. $4 \mathrm{~b}$ are compared with the plane cross section results shown in Fig. 3. The more precise analysis of matching of the two-dimensional FE model and 
the three-dimensional FE model is performed using the comparative diagrams shown in Fig. 5. The $y$ value in these diagrams corresponds to the vertical displacement from the upper edge of the acrylic block to any node in the finite element mesh in which the equivalent strain is reading. Figure 5a shows the corresponding diagrams for the results for equivalent Von Mises strains expressed in percent along the central line in the outer side of the acrylic block. The results shown in the diagram in Fig. 5a are then fitted with exponential function and the obtained curves and relations are given in Fig. $5 \mathrm{~b}$.

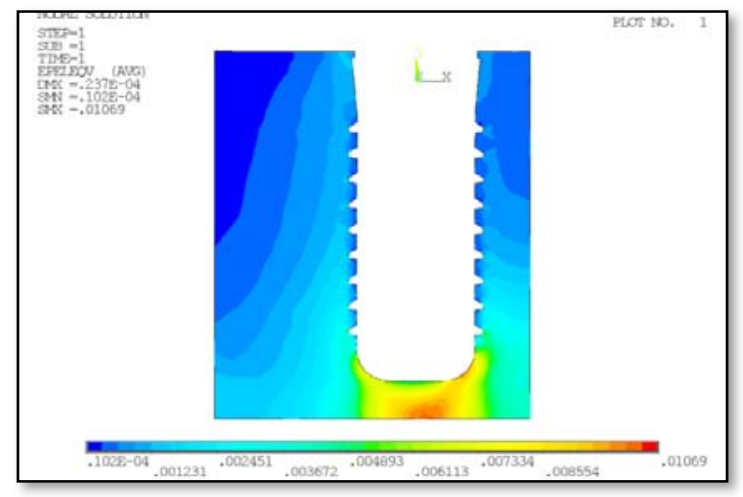

Fig. 3. Cross section plot for Equivalent Von Mises strain contours

The appropriate diagrams are created for FE models verification using experimental results, (Mitrovic et al. 2016, Tanasic et al. 2015). Figure 6 shows these diagrams.
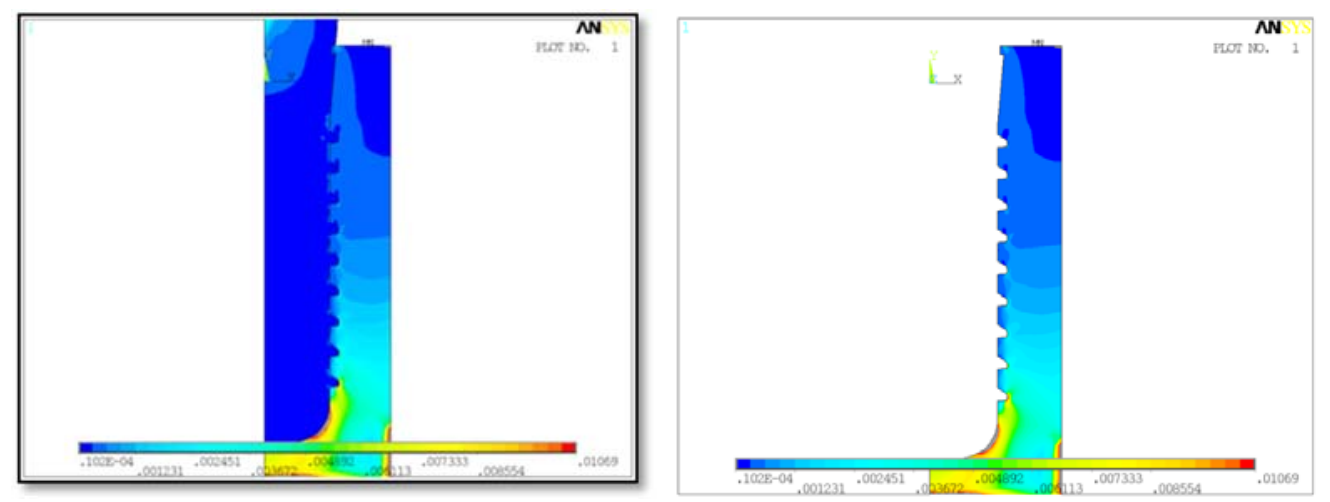

Fig. 4. Elastic Equivalent Von Mises strains for 2D FE model. (a) Implant and acrylic block; (b) Acrylic block 


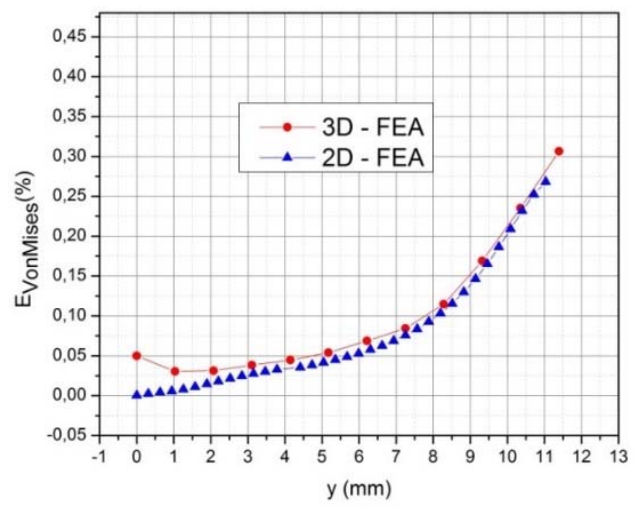

a)

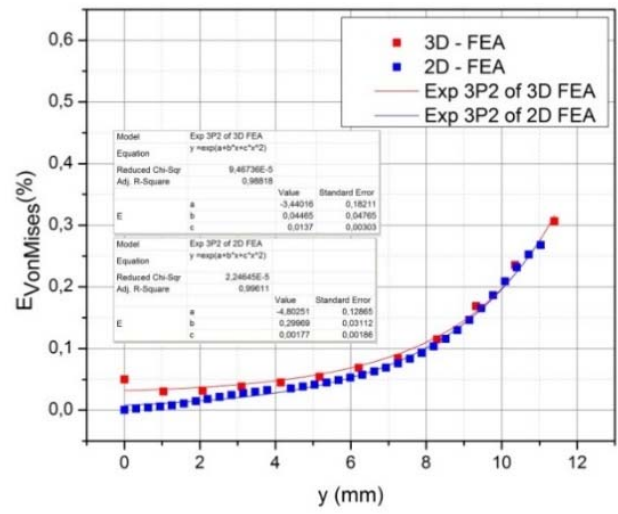

b)

Fig. 5. The comparative diagrams for two dimensional and three-dimensional results

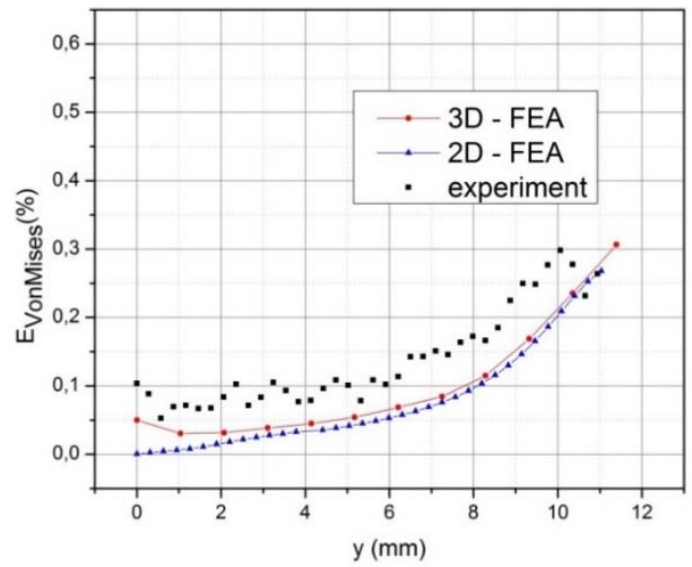

Fig. 6. The comparative diagrams for FE models' verification

\section{Numerical models for analysis of the effect of dental implant inclination}

Seven three-dimensional FE models of implant and acrylic block are developed in order to analyze the influence of implant inclination on deformations on the outer surface of acrylic block. The dimensions of the block model were $14.5 \times 13 \times 11.5$ $\mathrm{mm}$. The model of Strauman 4 x $12 \mathrm{~mm}$ cylindrical dental implant system (Straumann, Basel, Switzerland) (Table 1), was placed in the polymethyl methacrylate (Akrilat R, Galenika, Belgrade, Serbia) block with inclination of vertical axis in an interval of $(+1$ $\div-5$ ) degrees. The height of the block was $14.5 \mathrm{~mm}$, length $11.5 \mathrm{~mm}$ (profile view, Fig. 7). The width of the sample and the width of the surface of interest was $13 \mathrm{~mm}$. The axis of the implant was positioned symmetrically relative to the width of the area of the interest. The implant thread form was modeled as buttress with the pitch size of $1.25 \mathrm{~mm}$. 
The implant was placed so that his outer diameter surface was $2 \mathrm{~mm}$ away from the surface of interest, measured in the straight orientation $\left(0^{\circ}\right)$ (Fig. 7.). The inclination is modeled in respect to the intersection point of the vertical implant axis and the upper surface of acrylic block (Fig. 7). Positive inclination of the implant represents the case when the implant head was inclined away from the measured surface and negative when it was inclined towards it (Fig. 7). The model with no inclination is referred to as the straight model $\left(0^{\circ}\right)$. The model names and their respective inclinations are presented in Table 2 .

Figure 8 shows the series of FE models for the implant vertical axis inclination in interval of $(+1 \div-5)$ degrees. Structural solid three-dimensional degenerate brick elements without midside nodes are used for the implant and acrylic block material simulation. The contact surfaces between the implant and the acrylic block in which the implant is placed is defined as inherent and frictionless.

The applied load was axial in accordance with tha data published in Ueda et al. (2004), with intensity of $500 \mathrm{~N}$ (Fig. 1). The external static load of $500 \mathrm{~N}$ is defined in the opposite y direction, as force in the central node of the implant top surface (Fig. 1), and the appropriate displacement constraints are defined on the acrylic block in the same way for all modeled implant inclinations. The model was fixed from the front and back side of the block, and the base was partially supported in other two directions as shown in Fig. 1, according to the already published experimental report in Mitrovic et al. (2016). Figure 1 shows the external load and the area of interest. On the measured surface, along the implant axis, section line 0 was placed (Fig. 1). This area of interest is the closest to the implant, and is therefore considered that its deformations due to the implant loading best reflects the implant design characteristics published in Tanasic et al. (2015). The height of the sample is measured from the top of the block downwards, as shown in Fig. 7.

\begin{tabular}{lcc}
\hline & Young modulus [GPa] & Poisson ratio, $v$ \\
\hline PMMA & 1.3 & 0.35 \\
Titanium (Ti-6Al-4V), & 110 & 0.32 \\
Tiossi R. et al. (2012) & & \\
\hline
\end{tabular}

Table 1. Material properties used in the study 


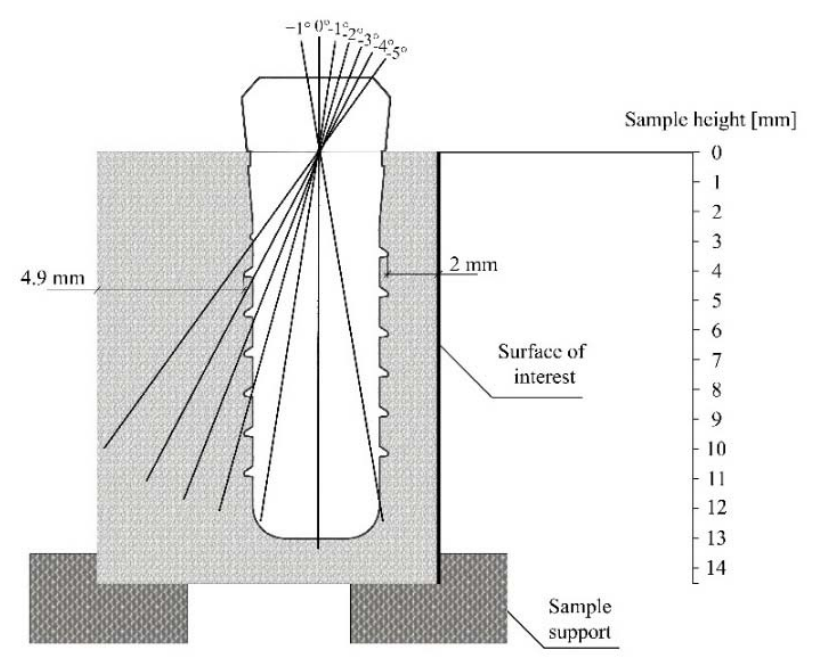

Fig. 7. Schematic representation of inclination for all seven models of dental implant

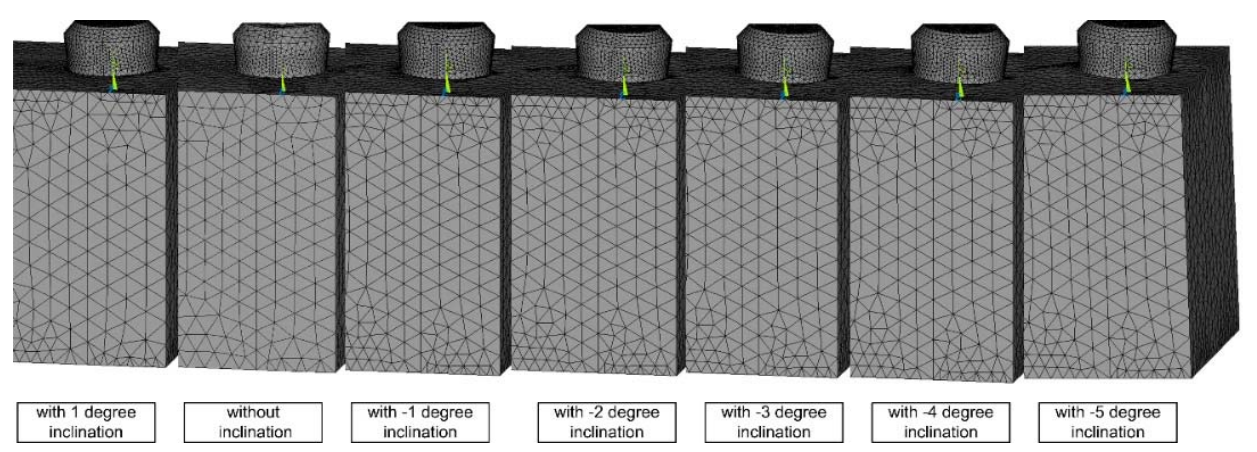

Fig. 8. Three - dimensional FE models for different implant inclination positions

\begin{tabular}{|c|c|}
\hline Model name & Inclination $\left[^{\circ}\right]$ \\
\hline Model 1 & +1 \\
Model 2 & 0 \\
Model 3 & -1 \\
Model 4 & -2 \\
Model 5 & -3 \\
Model 6 & -4 \\
Model 7 & -5 \\
\hline
\end{tabular}

Table 2. Model names and their relative inclinations

Finite Element Analysis results for strains were observed at two locations - block implant interface (cross section) and the area of interest. Horizontal strain is a deformation that occurs in the horizontal direction relative to the sample. Horizontal tensile strain implies elongation in horizontal directions, and compression implies compression in the horizontal direction of the sample. Also, vertical strain implies deformation in the vertical direction, or in a direction 
which is parallel with the dental implant axis. Vertical tensile strain indicates sample elongation in the vertical direction, while vertical compression implies compression in the vertical direction.

The cross section plots are obtained by plane cutting that passes through the implant center and is parallel with $x y$ coordinate plane, and they are used for the visualization of the influence of implant inclination on the strain state of the implant-block set. A comparative display of the obtained Elastic Equivalent Von Mises strains (VMs) for all modeled cases is used for analysis. For better comparison, obtained elastic equivalent Von Mises strains on the area of interest, are shown as contour plots. The elastic equivalent strains distribution has an expected layout. Each color in the results corresponds to the certain range of strain percentage, according to the color scale associated with it. The color scale, on strain field results, should be multiplied by 100 for strain percentage values. The diagrams are presented for first $12 \mathrm{~mm}$ of the sample height, which is also the implant height.

\section{Results}

The static Finite Element Analysis (FEA) is performed and the obtained strains interval is within the elasticity of modeled materials for all modeled cases of inclination. The results are displayed for the cross section and area of interest. In the cross section, only Von Mises strains (VMs) were presented. In the area of interest, Von Mises strain (VMs) (Fig. 10); horizontal strain (HBs) (Fig. 11) and vertical strain (VBs) (Fig. 12) were shown in the form of contour plots. Additionally, comparative diagrams were shown for all types of strains and for all the models used in this study (Figs. 13-14).

All results are shown only for the maximum load force of 500 N. The cross section (Fig. 9) displays strain concentration below the implant apex. The intensity of strain field was monitored by the color scale below Fig. 9 .

Figure 9 displays strain concentration below the implant apex. The area affected by the VMs (above 0.4\%) is the greatest for the models 6 and 7 near the implant apex.

The contour plot (Fig. 10) shows VMs distribution across the area of interest. The highest VMs observed in the area of interest are $0.4 \%$, located at the bottom of the block, below the implant apex. VMs of $0.2 \%$ are located in the upper portion of the block. The highest region under VMs can be found at model $7\left(-5^{\circ}\right)$. 


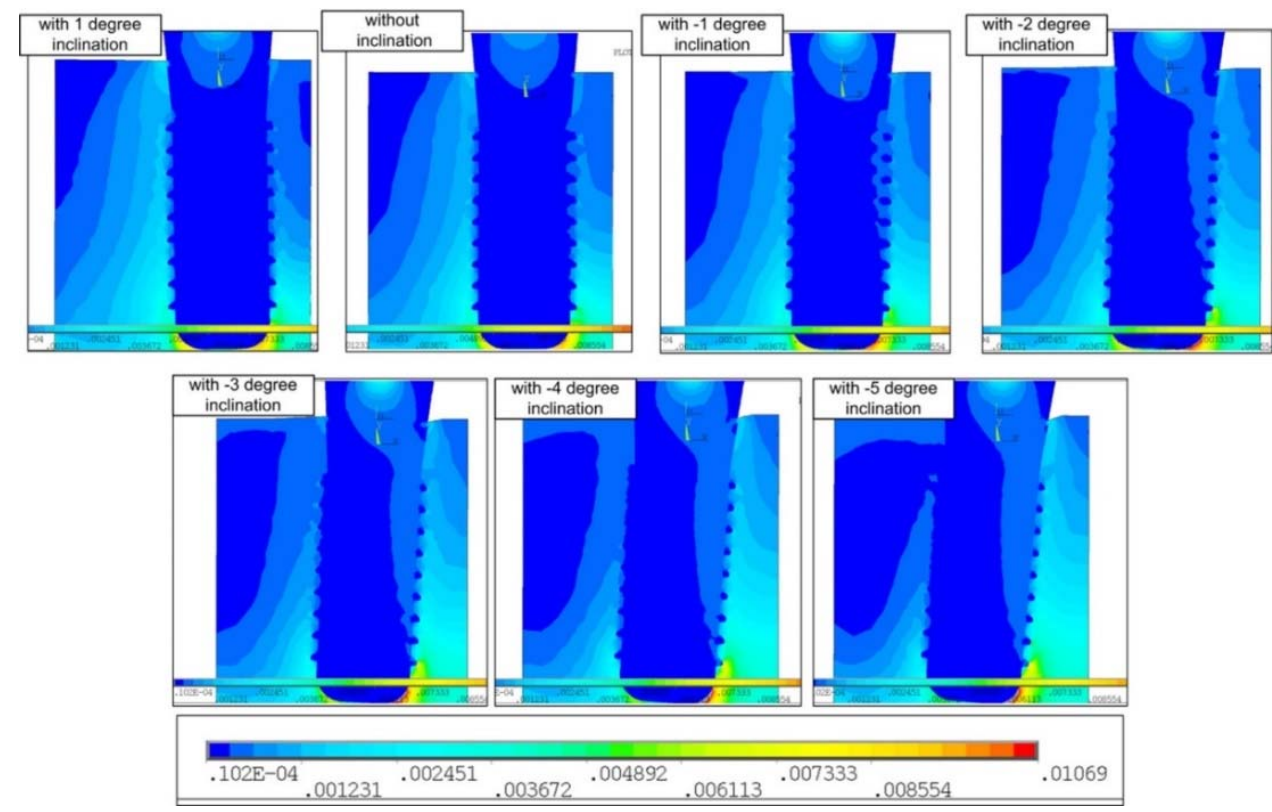

Fig. 9. Cross section plot for Equivalent Von Mises strains contours for different implant inclination positions

Figures 11 and 12 show HBs and VBs on the surface of interest, which are in this case mostly tensile and compressive, respectively.

For HBs field, compressional and tensile HBs occur. The highest compressive strain $(0.15$ $\%$ ) is located at the bottom of the positively inclined model $1\left(+1^{\circ}\right)$. This HBs is significantly lower for model $7\left(-5^{\circ}\right)$. Model 7 shows higher tensile HBs in the area of the implant apex, around $0.09 \%$. Greater inclination also brings higher tensile HBs in the area of the implant apex, and for model $7\left(-5^{\circ}\right)$, this is $0.09 \%$. 

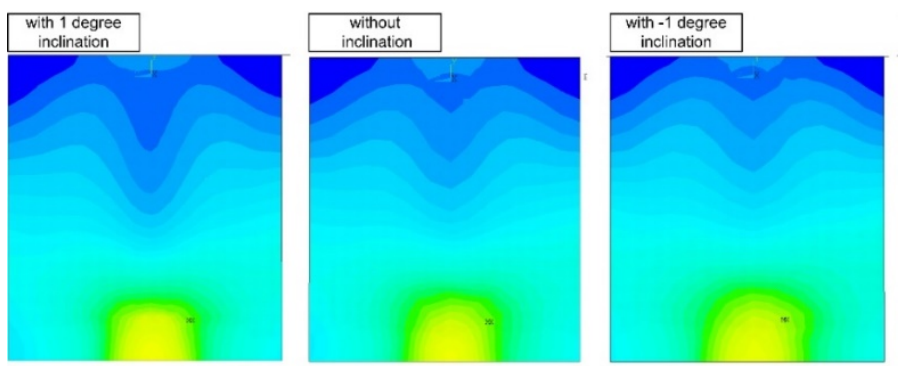

with -2 degree
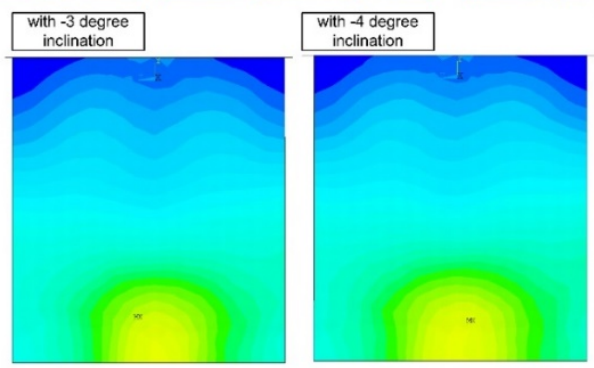

with -5 degree
inclination
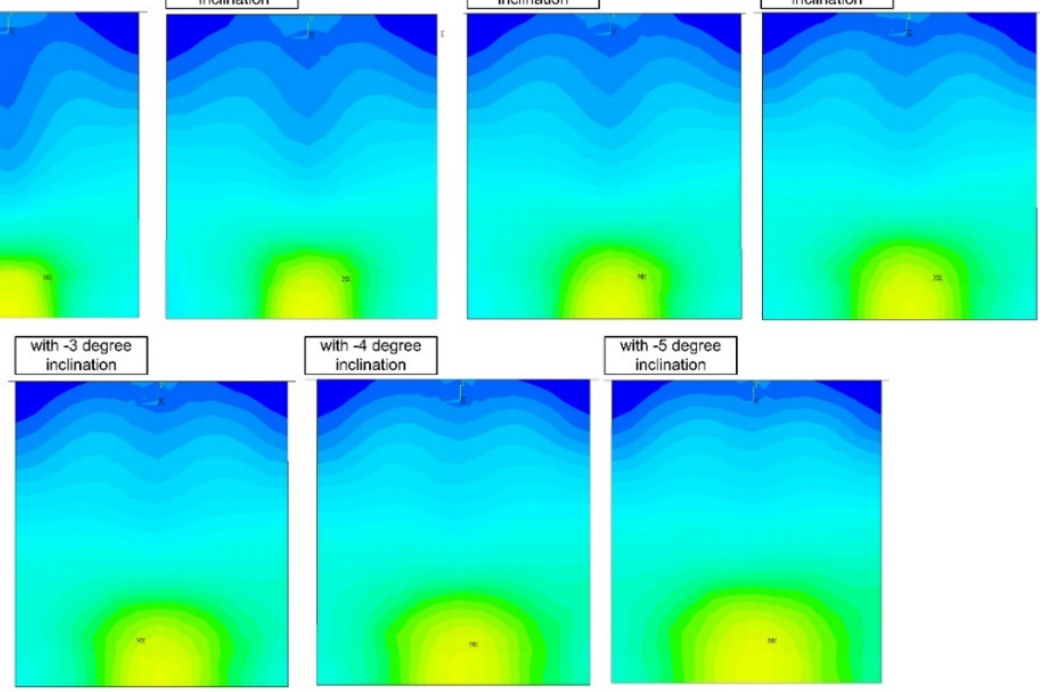

$.102 \mathrm{E}-04$

$695 \mathrm{E}-03^{.001379} .002064^{.002748}$

003433

004117

.004802

006

Fig. 10. Contour diagrams of Von Mises strain, for all models' surfaces
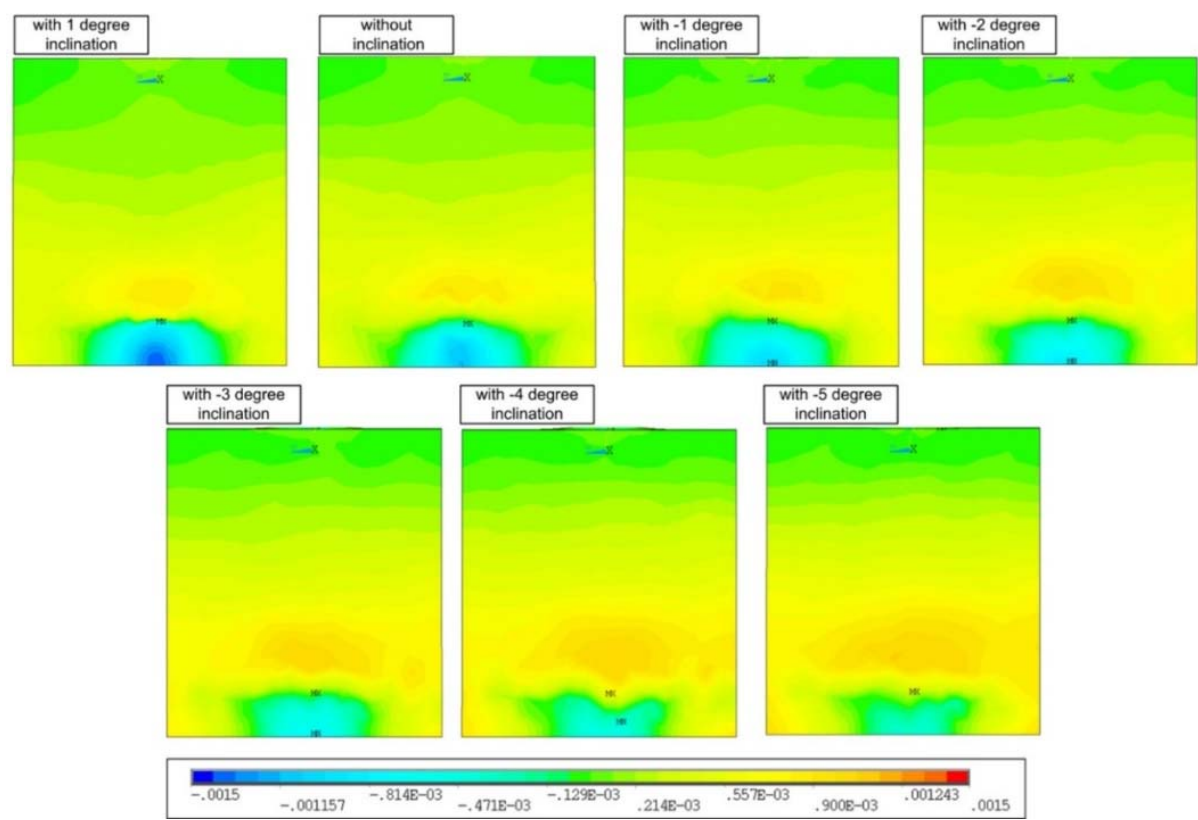

Fig. 11. Contour plot for $\mathrm{x}$ axis component strains for different implant inclination positions 

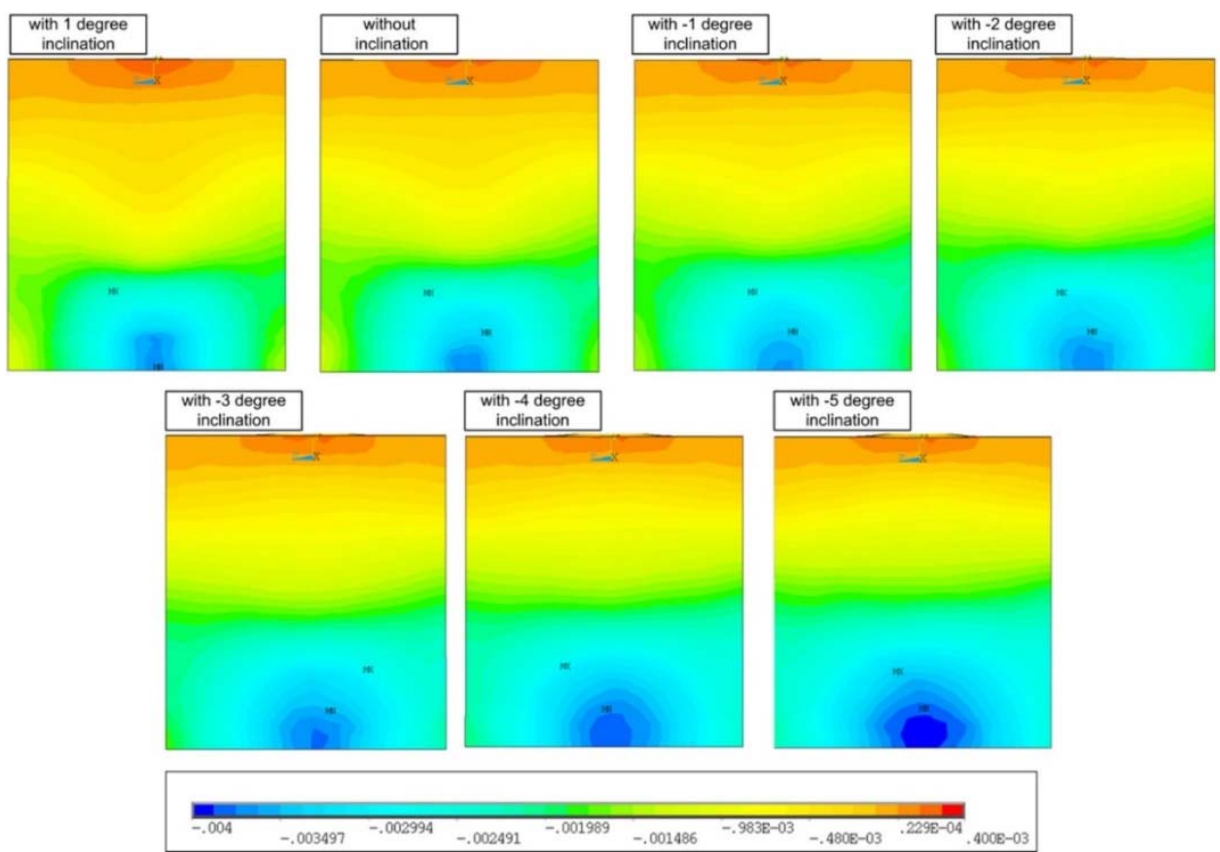

Fig. 12. Contour plot for y axis component strains for different implant inclination positions

\begin{tabular}{|c|c|c|c|c|c|c|c|}
\hline $\begin{array}{c}\text { Inclination } \\
{\left[{ }^{\circ}\right]}\end{array}$ & 1 & 0 & -1 & -2 & -3 & -4 & -5 \\
\hline $\begin{array}{c}\text { Sample } \\
\text { Height }\end{array}$ & & & & & & & \\
\hline 1.0357 & 0.0109 & 0.00659 & 0.00842 & 0.00556 & 0.00215 & 0.0031 & -0.00106 \\
\hline 2.0714 & 0.0143 & 0.0104 & 0.0126 & 0.0109 & 0.0106 & 0.00952 & 0.00982 \\
\hline 3.1071 & 0.0166 & 0.0134 & 0.0181 & 0.0181 & 0.0185 & 0.0206 & 0.0218 \\
\hline 4.1428 & 0.018 & 0.0154 & 0.0215 & 0.0228 & 0.0251 & 0.0283 & 0.0301 \\
\hline 5.1717 & 0.0206 & 0.0181 & 0.0251 & 0.0274 & 0.0324 & 0.0368 & 0.0399 \\
\hline 6.2142 & 0.0238 & 0.0221 & 0.0297 & 0.0355 & 0.0404 & 0.0443 & 0.0514 \\
\hline 7.2497 & 0.0292 & 0.0258 & 0.0382 & 0.0446 & 0.0497 & 0.0551 & 0.063 \\
\hline 8.2857 & 0.0374 & 0.0329 & 0.0513 & 0.056 & 0.0639 & 0.0716 & 0.0786 \\
\hline 9.3214 & 0.0578 & 0.0459 & 0.0678 & 0.0791 & 0.0845 & 0.0897 & 0.0957 \\
\hline 10.3571 & 0.0809 & 0.0622 & 0.0884 & 0.1001 & 0.1007 & 0.1063 & 0.1072 \\
\hline 11.3929 & 0.0849 & 0.0655 & 0.0902 & 0.0975 & 0.1001 & 0.1071 & 0.1041 \\
\hline 12.4286 & 0.0852 & 0.066 & 0.091 & 0.0982 & 0.1005 & 0.11 & 0.105 \\
\hline
\end{tabular}

Table 3. HBs ( $\varepsilon x)$ values for all models 


\begin{tabular}{|c|c|c|c|c|c|c|c|}
\hline $\begin{array}{c}\text { Inclina- } \\
\left.\text { tion [ }{ }^{\circ}\right]\end{array}$ & 1 & 0 & -1 & -2 & -3 & -4 & -5 \\
\hline $\begin{array}{c}\text { Sample } \\
\text { Height }\end{array}$ & & & & & & & \\
\hline 1.0357 & 0.0089 & $-8.03 \mathrm{E}-04$ & 0.0014 & $-1.16 \mathrm{E}-04$ & $-7.9 \mathrm{E}-04$ & -0.0043 & -0.00483 \\
\hline 2.0714 & -0.0109 & -0.0142 & -0.0181 & -0.0236 & -0.027 & -0.0316 & -0.0365 \\
\hline 3.1071 & -0.022 & -0.0264 & -0.0365 & -0.0442 & -0.0523 & -0.0607 & -0.0694 \\
\hline 4.1428 & -0.031 & -0.0342 & -0.0485 & -0.0575 & -0.0686 & -0.081 & -0.091 \\
\hline 5.1717 & -0.0382 & -0.0421 & -0.0605 & -0.0723 & -0.0855 & -0.1011 & -0.1146 \\
\hline 6.2142 & -0.0463 & -0.0523 & -0.0747 & -0.0908 & -0.107 & -0.1221 & -0.1404 \\
\hline 7.2497 & -0.0615 & -0.0641 & -0.0969 & -0.1163 & -0.136 & -0.1508 & -0.1711 \\
\hline 8.2857 & -0.086 & -0.0861 & -0.1322 & -0.149 & -0.1725 & -0.1917 & -0.2144 \\
\hline 9.3214 & -0.139 & -0.1235 & -0.1842 & -0.2042 & -0.2265 & -0.2455 & -0.2621 \\
\hline 10.3571 & -0.21 & -0.1702 & -0.2526 & -0.2705 & -0.2834 & -0.3011 & -0.3141 \\
\hline 11.3929 & -0.278 & -0.2157 & -0.3084 & -0.3214 & -0.3318 & -0.3413 & -0.3545 \\
\hline 12.4286 & -0.328 & -0.2345 & -0.3323 & -0.3383 & -0.3482 & -0.365 & -0.3761 \\
\hline
\end{tabular}

Table 4. VBs (عy) values for all models

VBs field (Fig. 12) is overall compressive, except the neck region of the block, where there are very low tensile strains of around $0.023 \%$. The highest compressive VBs are located at the bottom, from $0.4 \%$, for model $7\left(-5^{\circ}\right)$ to $0.35 \%$ for model $2\left(0^{\circ}\right)$. Also, model $7\left(-5^{\circ}\right)$ displays a greater area under maximum VBs (Fig. 7).

The diagram of VMs along section 0 is presented in Fig. 13. The height of the block is displayed on the horizontal axis and VMS on the vertical axis. Maximum VMs of around $0.4 \%$ are located at the height of the implant apex. This diagram enables easier tracking of block deformation across its height.

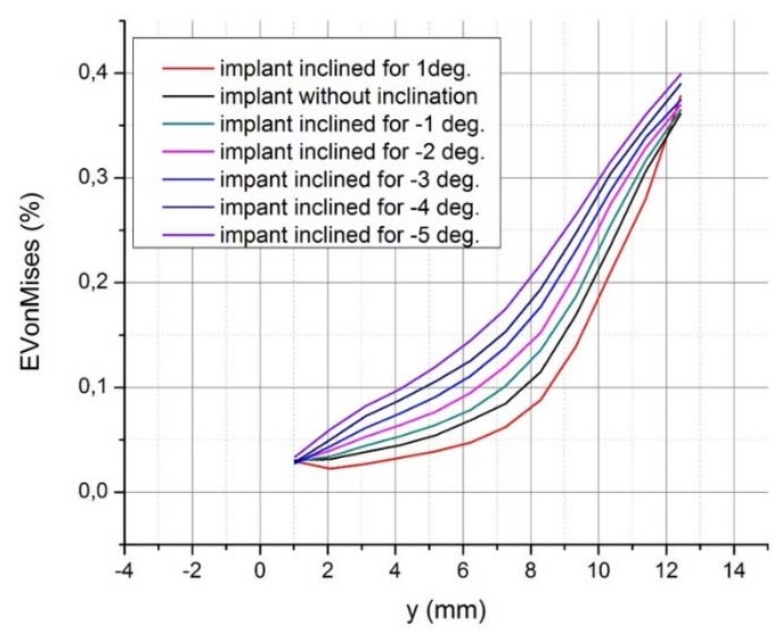

Fig. 13. Comparative diagram of Von Mises strains for all models. 


\begin{tabular}{|c|c|c|c|c|c|c|c|}
\hline $\begin{array}{c}\text { Inclination } \\
{\left[{ }^{\circ}\right]}\end{array}$ & 1 & 0 & -1 & -2 & -3 & -4 & -5 \\
\hline $\begin{array}{c}\text { Sample } \\
\text { Height }\end{array}$ & & & & & & & \\
\hline 1.0357 & 0.0292 & 0.0305 & 0.0292 & 0.0289 & 0.0273 & 0.0287 & 0.0335 \\
\hline 2.0714 & 0.0223 & 0.0314 & 0.0338 & 0.0396 & 0.0436 & 0.05 & 0.0597 \\
\hline 3.1071 & 0.0269 & 0.0382 & 0.0442 & 0.0527 & 0.0613 & 0.0723 & 0.082 \\
\hline 4.1428 & 0.0327 & 0.0447 & 0.0533 & 0.0638 & 0.0751 & 0.0879 & 0.0983 \\
\hline 5.1717 & 0.0388 & 0.0541 & 0.064 & 0.0764 & 0.0906 & 0.1056 & 0.1195 \\
\hline 6.2142 & 0.0473 & 0.0687 & 0.0785 & 0.0949 & 0.1109 & 0.1254 & 0.1448 \\
\hline 7.2497 & 0.0621 & 0.0844 & 0.101 & 0.1201 & 0.1383 & 0.153 & 0.1746 \\
\hline 8.2857 & 0.088 & 0.1148 & 0.1355 & 0.1521 & 0.1767 & 0.1938 & 0.2173 \\
\hline 9.3214 & 0.1389 & 0.1689 & 0.1864 & 0.2085 & 0.2306 & 0.2469 & 0.2645 \\
\hline 10.3571 & 0.21 & 0.2354 & 0.2551 & 0.2752 & 0.2879 & 0.3045 & 0.3156 \\
\hline 11.3929 & 0.28 & 0.3065 & 0.3166 & 0.3286 & 0.3389 & 0.3483 & 0.3603 \\
\hline 12.4286 & 0.3782 & 0.3612 & 0.3649 & 0.3696 & 0.3743 & 0.3896 & 0.3988 \\
\hline
\end{tabular}

Table 5. VMs values for all models

In Figures 14a and 14b, HBs and VBs along section 0 were presented, respectively. HBs were tensile along the implant height, growing gradually from the top of the block towards the implant apex. HBs value is highest at the implant apex, with $0.105 \%$ for model $7\left(-5^{\circ}\right)$.
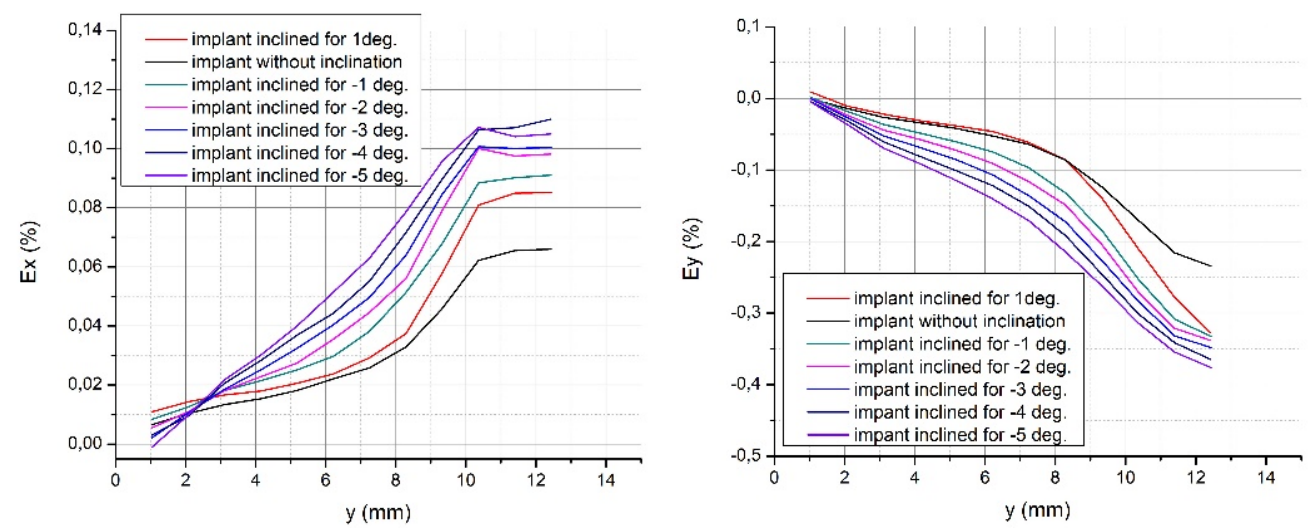

Fig. 14. The comparative diagrams for component strains for different implant inclination positions: a) $\mathrm{x}$ axis component strains; b) y axis component strains

\section{Discussion and conclusions}

Load transfer of inclined implant is frequently investigated in splinted configuration or implant supported fixtures (Behnaz et al. 2015, Gul and Suca 2014, Markarian and Ueda 2007, Cağlar et al. 2006, Almeida et al. 2013, Hong et al. 2012, Lan et al. 2010, Takahashi, Shimamura and Sakurai 2010, Ueda et al. 2004, Naini et al. 2011). The attempt of this numerical study is to 
examine the effect of inclination of the implant embedded in the acrylate block, in the straightforward arrangement. In this FE study, VMs, HBs $\left(\varepsilon_{\mathrm{x}}\right)$ and VBs $\left(\varepsilon_{\mathrm{y}}\right)$ are analyzed, on PMMA models, similar to the already published reports in Tanasić et al. (2015). The observed regions are block-implant interface and surface of interest (Fig.7). Seven finite element models, with different inclinations (Table 2), were created in order to examine the dental implant inclination effect on the bone implant interface, and on the surface of interest. A full contact between the implant and the block model was presumed. The results show that with greater implant inclination, increased deformations occur on the block implant interface and also on the surface of interest which is located $2 \mathrm{~mm}$ from the implant.

In seven models which are discussed in this paper, implant inclinations, which vary from $+1^{\circ}$ to $-5^{\circ}$ are relative to the block surface, as presented in Fig.7. The inclination of the implant away from the surface of interest is referred as positive, and towards the surface of interest is referred as negative. Model 2, or model with no inclination, is simply named as the straight model.

The contour plots (Fig.10) show that highest values of VMs are located at the bottom of the block, below the implant's apex. As the inclination changes towards more negative angle values, the point of maximal VMs in the block bottom is moving upwards. Another consequence of this inclination is that the area affected by this VMs $(0.4 \%)$ increases significantly.

Strain value increased along the section 0 as the inclination angles changed from positive to negative (Fig. 7, 11 and 12). VMs (Figs. 9-11) for all models converge to the maximum strain value of around $0.4 \%$, at the bottom of the sample. Comparative diagrams (Figs. 13-14) were introduced, so a more precise analysis of dental inclination influence on the strain values could be performed. Most optimal strain distribution was noticed for the positively inclined model (model 1). The least favorable strain distribution was observed when the greatest inclination was reached (model 7.).

Similar findings can be observed with HBs and VBs, where HBs are mostly tensile, and VBs compressive. HBs tensile show $59 \%$ more HBs on the model $7\left(-5^{\circ}\right)$ than model $2\left(0^{\circ}\right)$, in the area of the implant apex. Also, at the sample height of $8 \mathrm{~mm}$, model $7\left(-5^{\circ}\right)$ shows over 140 $\%$ higher tensile HBs than models $2\left(0^{\circ}\right)$.

VBs compressive are lowest at the top of the block, with the highest values around the implant apex $(12 \mathrm{~mm})$. Model $7\left(-5^{\circ}\right)$ shows $64 \%$ higher compressive VBs than for the same point at model $2\left(0^{\circ}\right)$. At $8 \mathrm{~mm}$, model $7\left(-5^{\circ}\right)$ has $156 \%$ higher compressive VBs than model 2 $\left(0^{\circ}\right)$ at the same height of the block- Maximum strains, for all models can be seen in the bottom region, around the implant apex. The source of this strain could be the reaction between the base and the sample, and that could be the reason why strain for models converges to the single value, in the lower region of the block. Above this region, higher strain values probably occur due to proximity of the implant apex, which correlates with the reports which state that low stresses and high strains usually occur at the implant apex, explained in Jian-Ping Geng P. et al. (2001) for all chancellors' models. Although the material used here is not the cancellous bone, it has similar characteristics with its Young modulus of $1.3 \mathrm{GPa}$ compared to $1.37 \mathrm{GPa}$ reported for the cancellous bone by Ding et al. (2009). This findings also agree with the other reports stating that having an inclined implant is less favorable for stress and strain distribution (Watanabe et al. 2003, Almeida et al. 2013, Lan et al. 2010). The contour plots for vertical strain (Fig. 12) show noticeably higher strain at the top of the block, near the implant neck, which is also reported (Watanabe et al. 2003, Hong et al. 2012).

Force intensity in this study was chosen so the strain in the implant vicinity would be more pronounced. It should be mentioned that this force intensity and higher are reported in the 
literature as the masticatory force, following Brunski (1984). Additionally, a change of inclination angles in this paper is smaller relative to the other reports (Watanabe et al. 2003, Almeida et al. 2013, Takahashi, Shimamura and Sakurai 2010). Nonetheless, it is reported that this incremental change does occur during the implantation procedure, due to surgeons' inexperience and other factors, following Payer et al. (2008), and effect of this errors should be widely known.

The Finite element analysis in this study used material properties which might not be sufficiently accurate for this simulation. However, these results are important because of the relative ratios between deformation and angle of inclination.

This paper explores the possibility of using acrylate models for acquiring knowledge about biomechanical load transfer. It should be noted that the aim of this study was not to report absolute values of strain, but to compare strain levels in different implant inclinations.

All the results are shown only for the maximum load force of $500 \mathrm{~N}$. The cross section (Fig. 9) displays strain concentration below the implant apex. The intensity of strain field was monitored by the color scale below Fig. 9.

Within the limitations of this study, it can be concluded that inclined implants lead to higher strains at the block-implant interface and this could also be observed on the area of interest. The points which are recognized as the region of best indication of implant inclination effect are located in the middle section of the implant body, at $7 \mathrm{~mm}$ for Von Mises strains, and $8 \mathrm{~mm}$ for horizontal and vertical strains. This point and the region around the implant apex in the area of interest could provide a clue about positive or negative effect of implant inclination. The results of this numerical model could be applied for the planning of future experimental studies which could utilize this and similar models to determine their load transfer characteristics. These insights can be useful in planning of dental implant position, and prediction of a successful dental therapy.

\section{Acknowledgements}

The authors acknowledge support the Ministry of Education, Science and Technological Development of Republic of Serbia, under Projects: OI174001, TR35031, TR35040 and TR32036.

\section{References}

Almeida EO et al. (2013). Tilted and Short Implants Supporting Fixed Prosthesis in an Atrophic Maxilla: A 3D-FEA Biomechanical Evaluation, Clin. Implant Dent. Relat. Res., 332-342. doi:10.1111/cid.12129.

Behnaz E et al. (2015). The effect of implant angulation and splinting on stress distribution in implant body and supporting bone: A finite element analysis, Eur. J. Dent. 9, 311. doi:10.4103/1305-7456.163235.

Brown SDK, Payne AGT (2011). Immediately restored single implants in the aesthetic zone of the maxilla using a novel design: 1-year report, Clin. Oral Implants Res. 22, 445-454. doi:10.1111/j.1600-0501.2010.02125.x.

Brunski J (1984). In vivo forces on endosteal implants: A measurement system and biomechanical considerations, 51 . 
Cağlar et al. (2006). Effects of mesiodistal inclination of implants on stress distribution in implant-supported fixed prostheses, Int. J. Oral Maxillofac. Implants. 21, 36-44. http://europepmc.org/abstract/MED/16519180.

Ding et al. (2009). Implant-bone interface stress distribution in immediately loaded implants of different diameters: a three-dimensional finite element analysis, J. Prosthodont. 18, 393402. doi:10.1111/j.1532-849X.2009.00453.x.

Djebbar $\mathrm{N}$ et al. (2010). Analysis of the effect of load direction on the stress distribution in dental implant, Mater. Des. 31, 2097-2101. doi:10.1016/j.matdes.2009.10.042.

Gul BE and Suca GC (2014) Finite Element Stress Analysis of Overdentures Supported by Angled Implants, Merit Res. J. Med. Med. Sci. 2, 196-206.

Hong HR et al. (2012). Effect of implant position, angulation, and attachment height on periimplant bone stress associated with mandibular two-implant overdentures: a finite element analysis., Int. J. Oral Maxillofac. Implants. 27, e69-76.

http://www.ncbi.nlm.nih.gov/pubmed/23057045.

Jian-Ping Geng P et al. (2001). Application of finite element analysis in implant dentistry: A review of the literature.

John A, Hobkirk, BDSand Thomas K Havthoulas (1998). The influence of mandibular deformation, implant numbers, and loading position on detected forces in abutments supporting fixed implant superstructures, J. Prosthet. Dent. 80, 169-174.

Lan T-H et al. (2010). Bone stress analysis of various angulations of mesiodistal implants with splinted crowns in the posterior mandible: a three-dimensional finite element study, Int. J. Oral Maxillofac. Implants. 25, 763-770. http://europepmc.org/abstract/MED/20657872.

Markarian RA, Ueda C (2007). Stress Distribution after Installation of Fixed Frameworks with Marginal Gaps over Angled and Parallel Implants: A Photoelastic Analysis, J. Prosthodont. 16, 117-122. doi:10.1111/j.1532-849X.2006.00161.x.

McCormick N, Lord J (2010). Digital Image Correlation, Mater. Today. 13, 52-54. doi:10.1016/S1369-7021(10)70235-2.

Mitrovic N et al. (2011). Application and Mode of Operation of Non-Contact Stereometric Measuring System of Biomaterials, FME Trans., 55-60.

Mitrovic N et al. (2016). Analysis of the effect of implant distance from the surrounding structure, in the PMMA block model, 21st European Conference on Fracture, ECF21, 2024 June 2016, Catania, Italy, Procedia Structural Integrity 2, 1260-1265.

Naini RB et al. (2011). Tilted or parallel implant placement in the completely edentulous mandible? A three-dimensional finite element analysis, Int. J. Oral Maxillofac. Implants. 26, 776-781. http://europepmc.org/abstract/MED/21841987.

Payer $\mathrm{M}$ et al. (2008). Surgical factors influencing mesiodistal implant angulation, Clin. Oral Implants Res. 19, 265-270. doi:10.1111/j.1600-0501.2007.01464.x.

Sljivic $M$ et al. (2016). Implemenation of FEM and Rapid Prototyping in Maxillofacial Surgery. FME Trans 44.4, 422-429.

Takahashi T, Shimamura I, Sakurai K (2010). Influence of number and inclination angle of implants on stress distribution in mandibular cortical bone with All-on-4 Concept, $J$. Prosthodont. Res. 54, 179-184. doi:10.1016/j.jpor.2010.04.004.

Tanasic I et al. (2012a). Analysis of the compressive strain below the removable and fixed prosthesis in the posterior mandible using a digital image correlation method., Biomech. Model. Mechanobiol. 11, 751-8. doi:10.1007/s10237-011-0348-5.

Tanasić I et al. (2012b). Optical aspect of deformation analysis in the bone-denture complex., Coll. Antropol. 36, 173-8. http://www.ncbi.nlm.nih.gov/pubmed/22816217.

Tanasić I et al. (2015). An attempt to create a standardized (reference) model for experimental investigations on implant's sample, Measurement 72, 37-42. doi:10.1016/j.measurement.2015.04.029. 
Tian K et al. (2012). Angled abutments result in increased or decreased stress on surrounding bone of single-unit dental implants: a finite element analysis., Med. Eng. Phys. 34, 152631. doi:10.1016/j.medengphy.2012.10.003.

Tiossi R et al. (2012). Digital image correlation analysis on the influence of crown material in implant-supported prostheses on bone strain distribution., J. Prosthodont. Res. 56, 25-31. doi:10.1016/j.jpor.2011.05.003.

Ueda $\mathrm{C}$ et al. (2004). Photoelastic analysis of stress distribution on parallel and angled implants after installation of fixed prostheses., Braz. Oral Res. 18, 45-52. doi:10.1590/S180683242004000100009.

Watanabe F et al. (2003). Finite element analysis of the influence of implant inclination, loading position, and load direction on stress distribution, Odontology 91, 31-36. doi:10.1007/s10266-003-0029-7. 